\title{
Type B mandibuloacral dysplasia with congenital myopathy due to homozygous ZMPSTE24 missense mutation
}

\author{
Rabah Ben Yaou ${ }^{1,2,3,10}$, Claire Navarro ${ }^{4,10}$, Susana Quijano-Roy ${ }^{1,2,5}$, Anne T Bertrand ${ }^{1,2}$, Catherine Massart ${ }^{1,2}$, \\ Annachiara De Sandre-Giovannoli ${ }^{4}$, Juan Cadiñanos ${ }^{6,9}$, Kamel Mamchaoui ${ }^{1,2}$, Gillian Butler-Browne ${ }^{1,2}$, \\ Brigitte Estournet $^{5}$, Pascale Richard ${ }^{7}$, Annie Barois ${ }^{5}$, Nicolas Lévy ${ }^{4,8}$ and Gisèle Bonne $e^{\star, 1,2,7}$
}

\begin{abstract}
Mutation in ZMPSTE24 gene, encoding a major metalloprotease, leads to defective prelamin A processing and causes type B mandibuloacral dysplasia, as well as the lethal neonatal restrictive dermopathy syndrome. Phenotype severity is correlated with the residual enzyme activity of ZMPSTE24 and accumulation of prelamin A. We had previously demonstrated that a complete loss of function in ZMPSTE24 was lethal in the neonatal period, whereas compound heterozygous mutations including one PTC and one missense mutation were associated with type B mandibuloacral dysplasia. In this study, we report a 30-year longitudinal clinical survey of a patient harboring a novel severe and complex phenotype, combining an early-onset progeroid syndrome and a congenital myopathy with fiber-type disproportion. A unique homozygous missense ZMPSTE24 mutation (c.281T > C, p.Leu94Pro) was identified and predicted to produce two possible ZMPSTE24 conformations, leading to a partial loss of function. Western blot analysis revealed a major reduction of ZMPSTE24, together with the presence of unprocessed prelamin A and decreased levels of lamin A, in the patient's primary skin fibroblasts. These cells exhibited significant reductions in lifespan associated with major abnormalities of the nuclear shape and structure. This is the first report of MAD presenting with confirmed myopathic abnormalities associated with ZMPSTE24 defects, extending the clinical spectrum of ZMPSTE24 gene mutations. Moreover, our results suggest that defective prelamin A processing affects muscle regeneration and development, thus providing new insights into the disease mechanism of prelamin A-defective associated syndromes in general. European Journal of Human Genetics (2011) 19, 647-654; doi:10.1038/ejhg.2010.256; published online 26 January 2011
\end{abstract}

Keywords: ZMPSTE24; mandibuloacral dysplasia; congenital myopathy; prelamin A; secondary laminopathies

\begin{abstract}
INTRODUCTION
ZMPSTE24 (also known as FACE-1) encodes a ubiquitously expressed zinc metalloprotease. Prelamin A, the lamin A precursor, is the only known substrate of ZMPSTE24 in mammals. ${ }^{1}$ ZMPSTE24 is involved in post-translational proteolytic cleavage of carboxy terminal residues of farnesylated prelamin A to form mature lamin A. ${ }^{2}$ Prelamin A is initially farnesylated on the last cysteine within the prenylation 'CaaX' motif at the C-terminus end of the protein by a farnesyltransferase. ${ }^{3}$ Thereafter, ZMPSTE24 or RCE1 ${ }^{4}$ removes the last three residues 'aaX', before a carboxy-methyl group is added by the methyl transferase ICMT on the last remaining cysteine. ${ }^{5}$ Finally, a second cleavage is specifically carried out by ZMPSTE24, removing the last 15 amino acids. ${ }^{6}$ The product of this post-translational maturation pathway, mature lamin $\mathrm{A}$, is located both at the nuclear lamina and the nucleoplasm. A-type lamins A and C are expressed in all vertebrate differentiated cells, and are translated from alternatively spliced transcripts of the LMNA gene. After post-translational modifications,
\end{abstract}

A-type lamins assemble with B-type lamins to form the nuclear lamina, a filamentous meshwork underlying the inner nuclear membrane. ${ }^{7}$

Although LMNA mutations are known to cause several phenotypes named 'laminopathies' (OMIM $\left.{ }^{\star} 150330\right)$ involving striated muscles, adipose, nervous, cutaneous tissues and bone in an isolated or combined manner, ${ }^{7,8}$ the responsibility of ZMPSTE24 gene mutations (OMIM $\left.{ }^{*} 606480\right)$ in human disease has also been recognized. By a candidate gene strategy resulting from phenotype similarities, various homozygous or compound heterozygous ZMPSTE24 mutations were identified in two different and extremely rare premature ageing disorders: restrictive dermopathy (RD, OMIM \#275210) and B-type mandibuloacral dysplasia (MADB, OMIM \#608612). ${ }^{9-11} \mathrm{RD}$, the most severe disorder, is a lethal neonatal genodermatosis ${ }^{12}$ characterized by intrauterine growth retardation, tight, rigid and easily eroded skin with prominent superficial vessels, bone mineralization defects, dysplastic clavicles, arthrogryposis and early neonatal death.

${ }^{1}$ Inserm, UMRS_974, Paris, France; ${ }^{2}$ Université Pierre et Marie Curie, Paris 6, UM 76, CNRS, UMR 7215, Institut de Myologie, IFR14, Paris, France; ${ }^{3}$ Association Institut de Myologie, GH Pitié-Salpêtrière, Paris, France; ${ }^{4}$ Université de la Méditerranée, Inserm, UMR_S910, Génétique Médicale et Génomique Fonctionnelle, Faculté de Médecine de Marseille, Marseille, France; ${ }^{5}$ AP-HP, Hôpital Raymond Poincaré, Service de Neuropédiatrie, Garches, France; ${ }^{6}$ Departamento de Bioquímica y Biología Molecular. Universidad de Oviedo, Oviedo, Spain; ${ }^{7}$ AP-HP, Groupe Hospitalier Pitié-Salpêtrière, U.F. Cardiogénétique et Myogénétique, Service de Biochimie Métabolique, Paris, France and ${ }^{8}$ AP-HM, Département de Génétique Médicale, Hôpital d'enfants Timone, Marseille, France

*Correspondence: Dr G Bonne, Inserm U974, Institut de Myologie, GH Pitié-Salpêtrière, 47 boulevard de l'Hôpital, 75651, Paris cedex 13, France.

Tel: +33 1421657 23; Fax: +33 1421657 00; E-mail: g.bonne@institut-myologie.org

${ }^{9}$ Current address: Instituto de Medicina Oncológica y Molecular de Asturias, Oviedo, Spain.

10These authors contributed equally to this work.

Received 7 October 2010; revised 7 December 2010; accepted 14 December 2010; published online 26 January 2011 
MADB, an autosomal recessive disorder, is characterized by skeletal abnormalities including mandible and clavicle hypoplasia, acroosteolysis of the terminal phalanges, mottled pigmentation, cutaneous atrophy and generalized lipodystrophy with metabolic syndrome such as insulin resistance, glucose intolerance, diabetes mellitus and hypertriglyceridemia. ${ }^{13}$ In both disorders, no skeletal muscle involvement was reported so far.

The lack of ZMPSTE24 protein or its activity in cell lines from ZMPSTE24-mutated patients resulted in misshapen nuclei containing abnormally aggregated lamin $\mathrm{A} / \mathrm{C}$ associated with accumulation of unprocessed prelamin A. ${ }^{10,11,14-16}$ Whereas the ZMPSTE24 mutations found in MADB encoded a protein with considerably reduced residual activity, the mutations found in RD led to null ZMPSTE24 activity, strongly suggesting a positive correlation between residual enzyme activity, accumulated prelamin A levels and the severity of the phenotype. ${ }^{17}$ In Zmpste24-knockout mice, severe growth retardation, skeletal abnormalities, including small mandible, lipodystrophy, cardiomyopathy and muscular dystrophy, were reported, as well as premature death, with these features being associated with a complete absence of mature lamin $\mathrm{A}$ and an exclusive production of prelamin A. ${ }^{18,19}$ More importantly, several studies have demonstrated that blocking prelamin A farnesylation using either farnesyl transferase inhibitors or a combination of statins and aminobisphosphonates could prevent prelamin A accumulation, extend longevity and ameliorate disease phenotype in Zmpste $24^{-1-}$ mice, ${ }^{4,20}$ leading to therapeutic trials in diseases associated with defective prelamin A processing.

In this study, we report a 30-year longitudinal follow-up of a patient carrying a new homozygous missense ZMPSTE24 mutation leading to partial loss of enzymatic activity. The resulting clinical phenotype goes beyond the expected progeroid features and includes skeletal muscle involvement, suggesting that defective prelamin A processing may also induce muscle disease.

\section{PATIENTS AND METHODS}

Patient clinical history was assembled from the detailed medical reports of the neuropediatric department, Raymond Poincare hospital, Assistance Publique Hôpitaux de Paris, Garches, France, where the patient was followed up her whole life. Our study complies with the ethical guidelines of all the institutions involved.

Genetic analysis was carried out on DNA samples from peripheral blood lymphocytes of the patient, her non-affected parents, two sisters and one brother after informed consent. LMNA and ZMPSTE24 gene screening was carried out as described previously. ${ }^{10,21}$ The presence of ZMPSTE24 mutation (c.281T >C) was confirmed by enzymatic digestion of the PCR product using BslI (Ozyme, Saint-Quentin-en-Yvelines, France). ZMPSTE24 conformations were predicted in silico using the two algorithms TMPred (http://www.ch. embnet.org/software/TMPREDform.hml) and TMHMM (http://www.cbs.dtu. $\mathrm{dk} /$ services/TMHMM-2.0/).

Primary skin fibroblasts were obtained from cultured skin biopsies of the patient and of a normal control subject according to Muchir et al. ${ }^{22}$

Western blot analysis was carried out from a volume of $40 \mu \mathrm{g}$ of protein homogenates on 10\% SDS-PAGE for both control and patient primary skin fibroblasts. ZMPSTE24 and lamins $\mathrm{A}$ and $\mathrm{C}$ were detected using monoclonal anti-ZMPSTE24 antibody (205-8C10, Daiichi Chemical, Toyama, Japan $)^{11}$ and polyclonal anti-lamin A/C antibody (H-110, Santa Cruz Biotechnologies, CA, USA)..$^{21}$

Immunofluorescence analysis of lamins $\mathrm{A} / \mathrm{C}$ or prelamin $\mathrm{A}$ was then carried out using polyclonal anti-lamin A/C (both at 1/100, Santa Cruz Biotechnologies) and FITC-conjugated anti-rabbit (1/500, Santa Cruz Biotechnologies) or Alexa-568 anti-goat (1/500, Invitrogen, Cergy Pontoise, France), whereas emerin was detected by monoclonal MANEM5 anti-emerin (1/50, from G Morris, NEWI, UK) and Alexa-568 anti-mouse (1/500, Invitrogen).

Lifespan analysis of fibroblasts from the patient and from an age-matched healthy control was carried out as previously described. ${ }^{23}$ Cell populations were serially passaged until they stopped dividing. At cell isolation, all cell populations were considered to be at 1 mean population doubling. The number of population doublings at every passage was calculated as $\log (N / n) / \log 2$, where $N$ is the number of cells at the time of passage divided by the number of cells initially seeded $(n)$.

\section{RESULTS}

\section{Clinical phenotype}

The patient is the fourth daughter born to second-degree related Portuguese parents. There were no other antecedents in the family. Hydramnios was detected intrautero and delivery was premature at 35 weeks of gestational age. Birth weight was $1.715 \mathrm{~kg}$ ( -3 s.d.), length was $45 \mathrm{~cm}$ ( -4 s.d.) and head circumference was $29.5 \mathrm{~cm}$ ( -4 s.d.), indicating intrauterine growth retardation. Apgar test was normal but two episodes of cyanosis were noted during the first day of life. Joint contractures were observed, including at the ankles, knees (popliteal angle $=90^{\circ}$ ), hips and elbows. Skin was dysmature, with atrophic, dried, crumpled and desquamated hyperkeratotic aspect. Dysmorphic features with retrognathia were also associated. Karyotype was normal. Polycythemia (hematocrit $=63.1 \%$ ) and thrombocytosis (thrombocyte count $=514000 / \mathrm{mm}^{3}$ ) were also present at birth. Although mild axial and limb hypotonia was noted by the age of 3 months, motor milestones were within normal ranges. She walked independently at 17 months. Difficulty in walking, climbing stairs or rising from the floor appeared by the age of 2 years. Electromyography revealed myopathic patterns on deltoid, flexor carpi radialis and tibialis anterior muscles without spontaneous activities. Nerve conduction velocities were normal. Muscle weakness predominant in periscapular regions and proximal lower limbs progressed especially from the age of 4 years. There was scapular winging (Figure $1 a_{3-5}$ ). A deltoid muscle biopsy performed at 9 years revealed myopathic changes suggestive of fiber-type disproportion. There was fiber size variation with a large number of small fibers and type I fiber predominance (Figure $1 d_{1}$ ). All features were suggestive of a congenital myopathy with fiber-type disproportion. In the second decade, muscle deficit spread to the four limbs. At 26 years, muscle weakness extended rapidly to distal lower limbs, and electromyography examination revealed perineal nerve involvement in addition to a myopathic pattern at needle electromyography.

The patient demonstrated a severe failure to thrive (Figure 1a), which led to 'harmonic' dwarfism (Figure $1 \mathrm{a}_{1-5}$ ). Varied and severe complications were observed during the course of the disease, involving bones, skin, joints, skeletal, respiratory and cardiac muscles, and vascular, renal and hormonal systems. However, cognition was normal; she attended school, drove an adapted car and worked as an accountant until the age of 25 years.

Bone involvement was an early complication. Hypoplasia of the mandibula (Figure $1 c_{1}$ ) and left clavicle (Figure $1 b_{1-4}$ ) was detected by 5 months. Enlarged anterior fontanelle, delayed skull ossification and tooth eruption (Figure $1 c_{2}$ ) were noted at 9 months, but after 4 years teeth rapidly crowded (Figure $1 c_{3}$ ). Other bone abnormalities included femoral head dysplasia, osteolysis, delayed bone maturation and distal finger osteolysis (Figure $1 c_{5-6}$ ). Long bones had characteristic enlarged metaphysis and slender diaphysis (Figure $1 c_{5}$ ). Decreased spine and limb bone density was associated with multiple spontaneous fractures, first observed in a clavicle at 6 years of age (Figure $1 b_{5-6}$ ), and later in ribs, vertebrae and femur (Figure $1 b_{7}$ ). At the age of 13 years, osteolysis of the third and fourth cervical vertebral spine process accompanied by $\mathrm{C} 3-\mathrm{C} 4$ and $\mathrm{C} 7-\mathrm{D} 1$ anterolisthesis occurred (Figure $1 b_{8}$ ). Cranial sutures were still open at 15 years of 

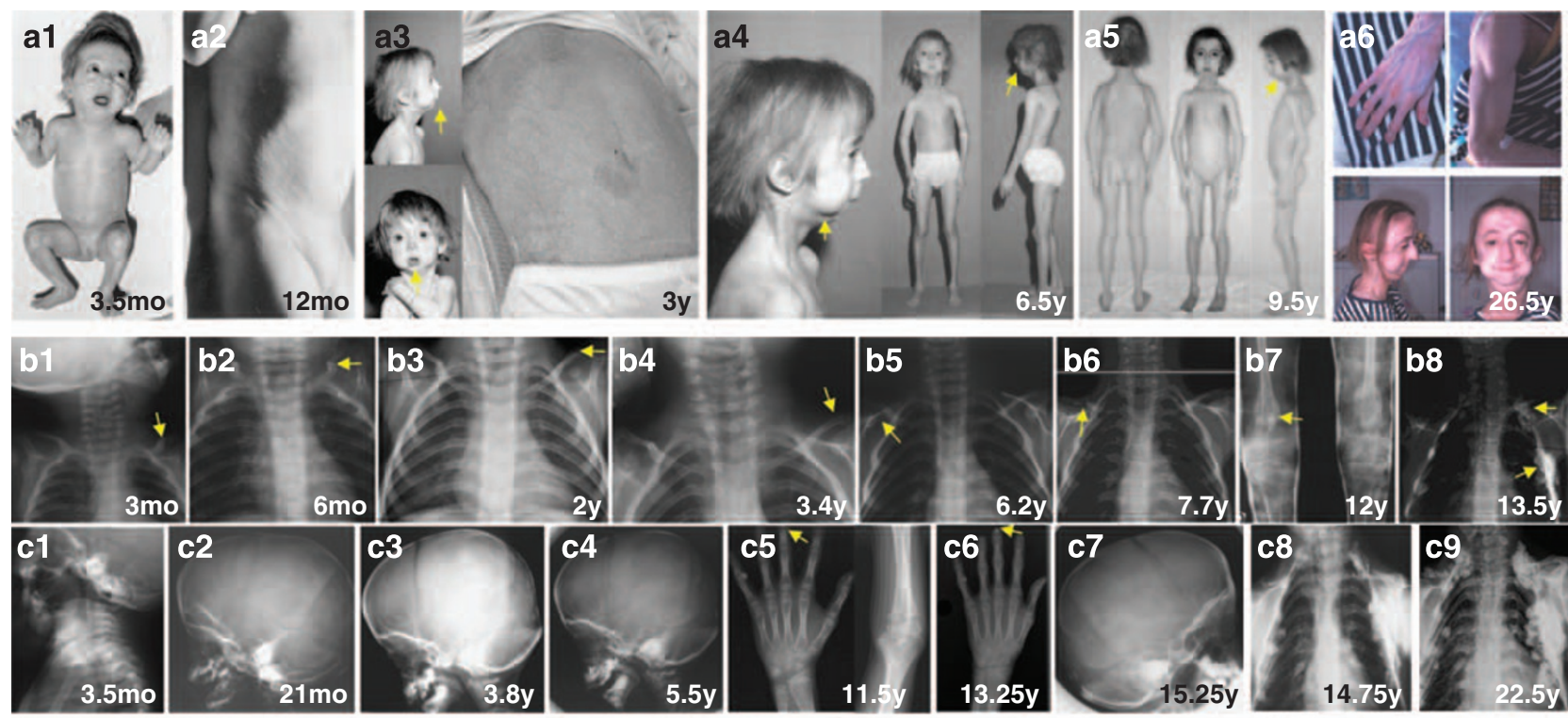

b8
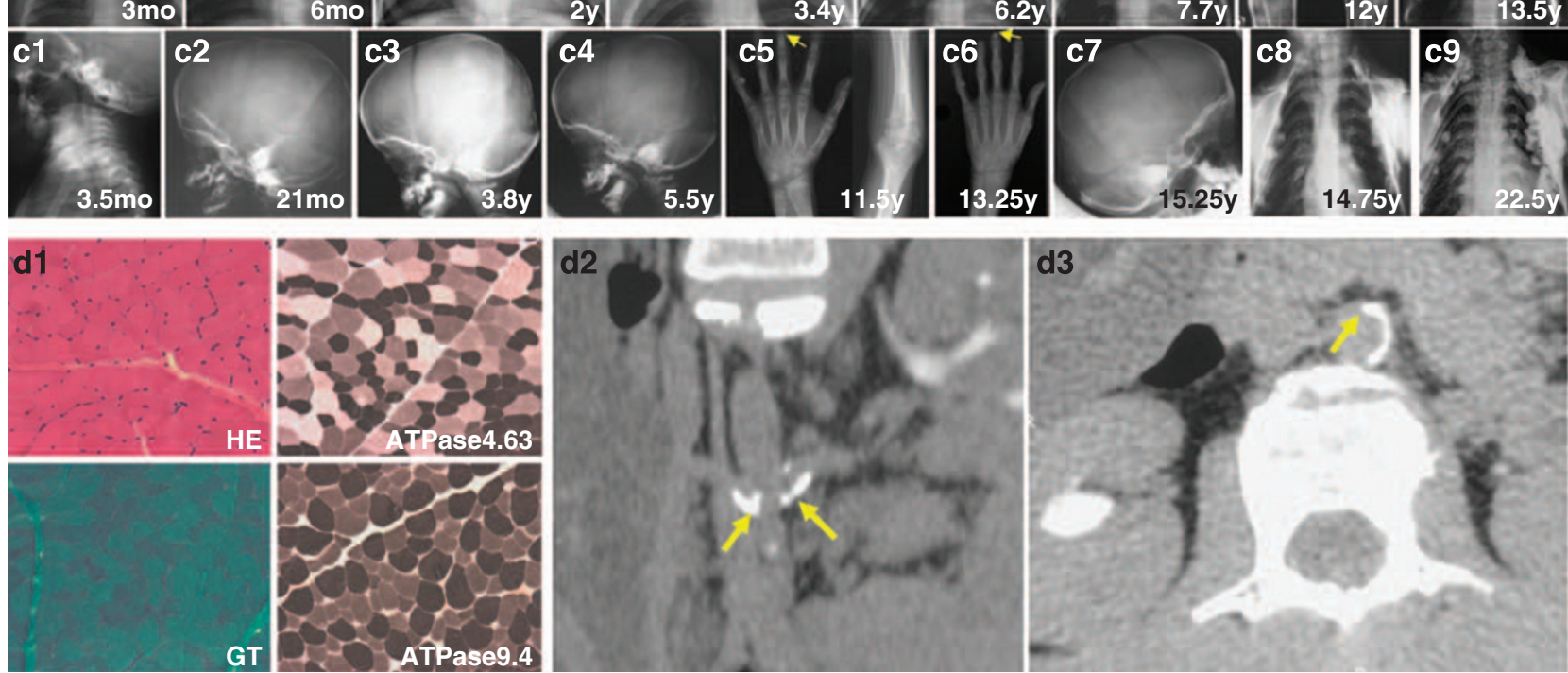

Figure 1 Patient clinical features. (a) Photographs of the patient at different ages showing general habitus, mandibular hypoplasia (a $3-6$, arrows) and abdominal skin papules with dry skin $\left(\mathbf{a}_{2}\right)$, pronounced abdominal $\left(\mathbf{a}_{3}\right)$ and hand $\left(\mathbf{a}_{6}\right)$ veins and alopecia ( $\left.\mathbf{a}_{6}\right)$. (b). Chest X-ray showing (arrows) dysplastic clavicles $\left(\mathbf{b}_{1-4}\right)$, right clavicle $\left(\mathbf{b}_{5-6}\right)$ and lower femur extremity $\left(\mathbf{b}_{7}\right)$ fractures, as well as subcutaneous cervical and dorsal calcifications $\left(\mathbf{b}_{8}\right)$. (c) Skull, hand

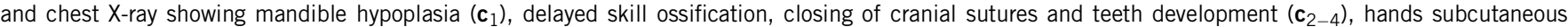
calcifications of the interphalangian joints with distal fingers acro-osteolysis $\left(\mathbf{c}_{5-6}\right)$, enlarged metaphysis with slender diaphysis of long bones ( $\left.\mathbf{c}_{5}\right)$ and diffuse subcutaneous cervical and dorsal calcifications $\left(\mathbf{c}_{8-9}\right)$. (d) Deltoid muscle biopsy ( $\mathbf{d}_{1}$ ) with hematoxylin-eosin (HE), modified Gomori trichrome (GT) and ATPase $\mathrm{pH} 4.6$ and 9.4 stainings showing fiber size variation, predominance of small type I fibers. CT scan coupled with angiography showing (arrows) bilateral calcifications at the renal arteries origin $\left(\mathbf{d}_{2}\right)$ and atheromatous aortic infiltration $\left(\mathbf{d}_{3}\right)$.

age (Figure $1 c_{7}$ ). Serum calcium, phosphate and alkaline phosphatase levels were normal since birth.

Cutaneous abnormalities typical of progeria, such as reduced subcutaneous fat, were noted since birth. Generalized sclerodermic skin and abdominal papules were observed during the first year (Figure $1 \mathrm{a}_{2}$ ). By the age of 14 months, frontal and abdominal veins were dilated (Figure $1 \mathrm{a}_{3}$ ) and the abdominal papules became beige and translucent. Later, blue spots appeared on the face and legs and a skin biopsy performed at the age of 9 years revealed sclerodermic features in the absence of deposits or inflammatory infiltrates. There was extreme dermal fibrosis, increased and often horizontally oriented elastic fibers, basal lamina horizontalization and rare dermal papules (data not shown). Subcutaneous calcifications appeared in different sites (interphalangian joints, cervical, dorsal and thoracic, Figure $\left.1 c_{8-9}\right)$. From the age of 13.5 years there was a rapid loss of hair evolving to alopecia in the year after (Figure $1 \mathrm{a}_{6}$ ).

The cervical paravertebral calcifications caused spine and chest rigidity, and other cervical subcutaneous nodules were probably responsible for swallowing difficulties observed at 15 years of age. By the age of 26 years, loss of muscle strength was predominant in lower limbs and a vertebral CT scan identified multiple subcutaneous and paravertebral calcifications at the cervicothoracic levels, infiltrating the left C7-T1 intervertebral foramen, the ribs and pushing back the scapula. Later, vertebral MRI and CT scan revealed two intracanalar calcifications, one reducing medullar cavity diameter and another causing medullar compression and infiltrating the intervertebral foramen. Surgical ablation of these calcifications led to good functional recovery. Joint contractures associated with the short clavicles resulted in chest flatness with protuberant right chondrocostal joints. Joint mobility became very limited during the course of the disease at the ankles, knees and hips, and a marked limitation of the mobility of shoulders and contractures of pectoral muscles was observed. With respect to the spine, by the age of 2 years, a mild dorsal kyphoscoliosis was noted and associated cervical and lumbar lordosis due to axial weakness. A Milwaukee brace was indicated at 6 years. By the age of 15.5 years, a dorsal gibbus was reported.

Respiratory complications were a late event but the patient died as a consequence of them. At 8 years, diurnal hypersomnia, dyspnea, nocturnal snoring and an impressive laryngeal stridor prompted the first respiratory evaluation. Forced vital capacity (FVC) was reduced to 
$40 \%$ of the theoretical value, with a significant fall in supine FVC with respect to sitting position, indicating a selective diaphragmatic failure and a major respiratory difficulty when lying. Polysomnographic evaluation revealed nocturnal apneas and marked desaturation. The patient was treated by non-invasive nocturnal ventilation. Theophylline was also started. Restrictive respiratory insufficiency was observed (FVC of $37 \%$ at 10 years and $28.2 \%$ at 25 years).

ECG and echocardiography were normal until 24 years, when sinusal tachycardia, sharpened $\mathrm{T}$ waves, right bundle branch block, ventricular extrasystols, concentric left ventricular hypertrophy and high blood pressure were observed. At 27 years, blood renin $(34.9 \mathrm{pg} / \mathrm{ml}, N<19)$ and aldosterone $(207 \mathrm{pg} / \mathrm{ml}, N<125)$ were elevated concomitantly with hypokalemia $(3.3 \mathrm{mmol} / \mathrm{l}, N>3.5)$. This prompted a CT scan of the renal arteries as well as MRI coupled with angiography, and revealed bilateral calcifications of the renal arteries (Figure $1 \mathrm{~d}_{2}$ ), resulting in a $50 \%$ reduction in diameter without renal parenchyma vascularization abnormalities. Atheromatous aortic infiltration was also observed (Figure $1 \mathrm{~d}_{3}$ ). High blood pressure was treated by acebutolol and enalapril with no significant improvement.

At 29 years, chronic kidney disease with severe anemia appeared and worsened progressively. Non-insulin-dependent diabetes mellitus was diagnosed but no treatment was administered. The last evaluation at 30 years showed elevated serum urea $(23 \mathrm{mmol} / \mathrm{l}, N<7.5)$, creatinine $(455 \mu \mathrm{mol} / \mathrm{l}, N<120)$ and phosphate $(2.73 \mathrm{mmol} / \mathrm{l}, \quad N<1.5)$ levels; the glomerular filtration rate was $9 \mathrm{ml} / \mathrm{mn}$ per $1.73 \mathrm{~m}^{2}$ $(N>80)$, proteinuria was $6.9 \mathrm{~g} / \mathrm{l}$, glycosuria was $2.5 \mathrm{~g} / \mathrm{l}$ and $\beta-2$ microglobinuria was $25000 \mathrm{mg} / 24 \mathrm{~h}$. MAG3 scan revealed a global reduction in tracer uptake and excretion, all suggestive of multifactor chronic kidney disease with glomerular, vascular and tubular abnormalities. Because of bilateral renal artery calcifications, a revascularization procedure was suggested. Meanwhile, hemodialysis and chronic peritoneal dialysis were also considered. Subsequently, at 30 years of age, in this context of severe renal insufficiency and high blood pressure, the patient developed a lung infection. During this episode, she felt unwell, went to bed and was found dead in her bed at home. No autopsy was performed.

Further investigations were carried out. Blood testing revealed additional abnormalities during the course of the disease. At 17 months, pre- $\beta$-lipoprotein levels were slightly elevated $(6.2 \mathrm{~g} / \mathrm{l}$, $N<6$ ). At 12 years, slight hearing loss was suspected at school. However, audiogram and auditory evoked potentials remained normal. Puberty was acquired at the age of 12.5 years without menarche or menstrual period anomalies. The patient had poor breast development. Hormonal evaluation at 13 years revealed reduced dehydroepiandrosterone $(1.6 \mathrm{ng} / \mathrm{ml}, N>2)$ and estradiol $(27 \mathrm{pg} / \mathrm{ml}$, $N>60)$, and elevated somatostatin $(28 \mathrm{pg} / \mathrm{ml}, \mathrm{N}<10)$, with the remaining hormonal parameters remaining normal (cortisol, deoxycortisols, progesterone, androstenedione, testosterone, corticotropin and prolactin). At 26.5 years, hypertriglyceridemia $(3.65 \mathrm{mmol} / \mathrm{l}$, $N<1.69)$, hypercholesterolemia $(7.54 \mathrm{mmol} / \mathrm{l}, N<5.2)$, hypertransaminemia (ALAT: $201 \mathrm{IU} / \mathrm{L}, N<35$; AST: $93 \mathrm{IU} / \mathrm{L}, N<34$ ) and increased gamma-GT $(244 \mathrm{IU} / \mathrm{L}, N<28)$ were observed. Abdominal ultrasonography revealed global hepatomegaly with steatosis with normal and symmetric ovaries. Medical treatment with simvastatin was started. CK levels were always within the normal range.

\section{Genetic analysis}

Because the main clinical features suggested a progeroid syndrome, with clinical features similar to MAD or HGPS, LMNA was first sequenced at the genomic level but no mutation was found in the exons or intron/exon boundaries. The ZMPSTE24 gene was subsequently sequenced and a homozygous variation (c.281T $>$ C) in exon 3, predicted to result in p.Leu94Pro, was identified (Figure 2a). This variation creates a new BslI restriction site that was further used to confirm the mutation in the patient and analyze its segregation within the family. A heterozygous c.281T $>$ C variation was identified in the non-affected parents, one sister and the brother, whereas it was absent in the other sister (Figure 2b). This variation was not detected in more than 200 control chromosomes from unrelated subjects. Partial alignment of the ZMPSTE24 protein sequences from various species showed that the mutated leucine 94 is highly conserved, suggesting an important role of this residue in enzymatic activity (Figure 2c). In silico predictions using TMHMM and TMpred programs, with either normal or mutated ZMPSTE24 protein sequences as matrix, indicated two possible conformational models: one corresponded to the normally folded protein including its seven consensus transmembrane (TM) domains, whereas the other lacked the second TM (Figure 2d). Consequently, this misfolded ZMPSTE24 predicted both its catalytic and endoplasmic reticulum (ER) signal mislocated in the ER lumen instead of cytoplasm, suggesting a loss of function or a mislocalization. These predictions could not be considered as being mutually exclusive.

\section{Western blot analysis}

To determine whether these ZMPSTE24 variations modify the expression of Zmpste24 protein, and consequently to prelamin A processing defects, western blot analyses were carried out on proteins extracted from the patient's cultured skin fibroblasts at passage P2. Immunoblots on the patient's fibroblast revealed an important decrease in the level of ZMPSTE24 compared with age-matched control fibroblasts. As expected, this reduction was associated with the accumulation of prelamin A; in addition to the wild-type lamins A and C, an extra band with higher molecular weight at the expected prelamin A size was observed (Figure 2e). This supplementary band was absent from control fibroblasts also analyzed at passage P2.

\section{Lifespan and expression of prelamin $\mathrm{A}$, lamin $\mathrm{A} / \mathrm{C}$ and emerin of fibroblasts}

The patient's fibroblasts were highly variable in size with a majority of the cells being larger than control cells. They tended to grow as clusters (Figure 3) and exhibited a reduced lifespan (Figure 4). Primary patient fibroblasts arrest their growth after only 56 days in culture (seven divisions). This is in contrast to primary control fibroblasts, which made 38 divisions after 130 days in culture (Figure 4). Patient fibroblasts exhibited major nuclear shape defects. Although lamins $\mathrm{A} / \mathrm{C}$ and emerin were still localized at the nuclear rim together with prelamin A in all patient's fibroblasts, severe nuclear shape defects (lobulations, herniations) were observed, as compared with the uniform oval or round nuclei in wild-type cells (Figure 3).

\section{DISCUSSION}

This is the first report describing a MADB phenotype associated with myopathic features caused by a homozygous missense ZMPSTE24 mutation. The pathogenicity of this unreported mutation was demonstrated by the presence of both prelamin A in protein extracts from patient fibroblasts, the abnormal shape of the fibroblast nuclei and the reduced lifespan of these fibroblasts.

In literature, 30 patients carrying ZMPSTE24 mutations have already been reported and have a large clinical spectrum going from typical RD to MADB. Apart from one, ${ }^{24}$ the 23 patients with typical $\mathrm{RD}$ features had either homozygous or compound heterozygous null mutations (nonsense, insertions or deletions) ${ }^{10,11,17,25-30}$ or very large 
a

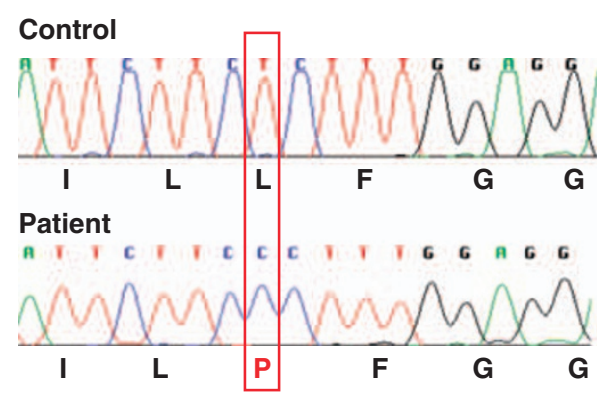

b

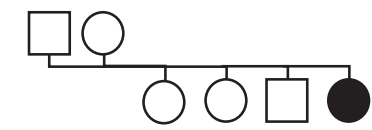

$+/ \mathrm{m}+/ \mathrm{m}+/ \mathrm{m}+/++/ \mathrm{m} \mathrm{m} / \mathrm{m}$

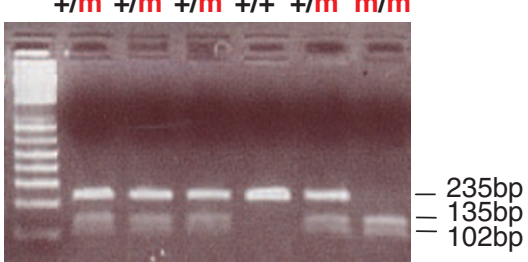

C

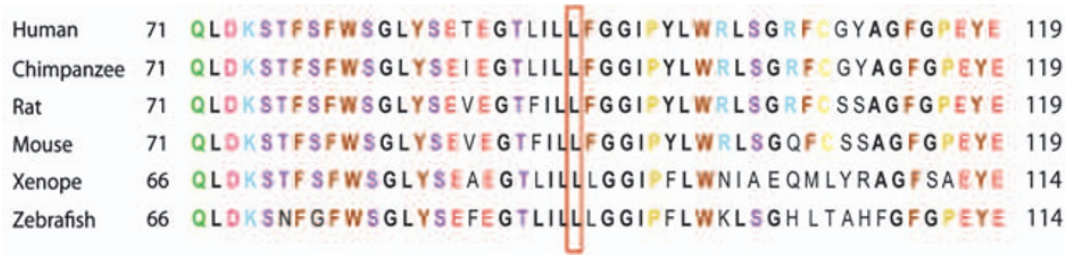

d
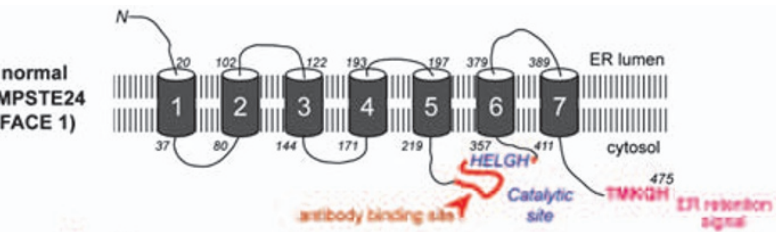

e (FACE 1)
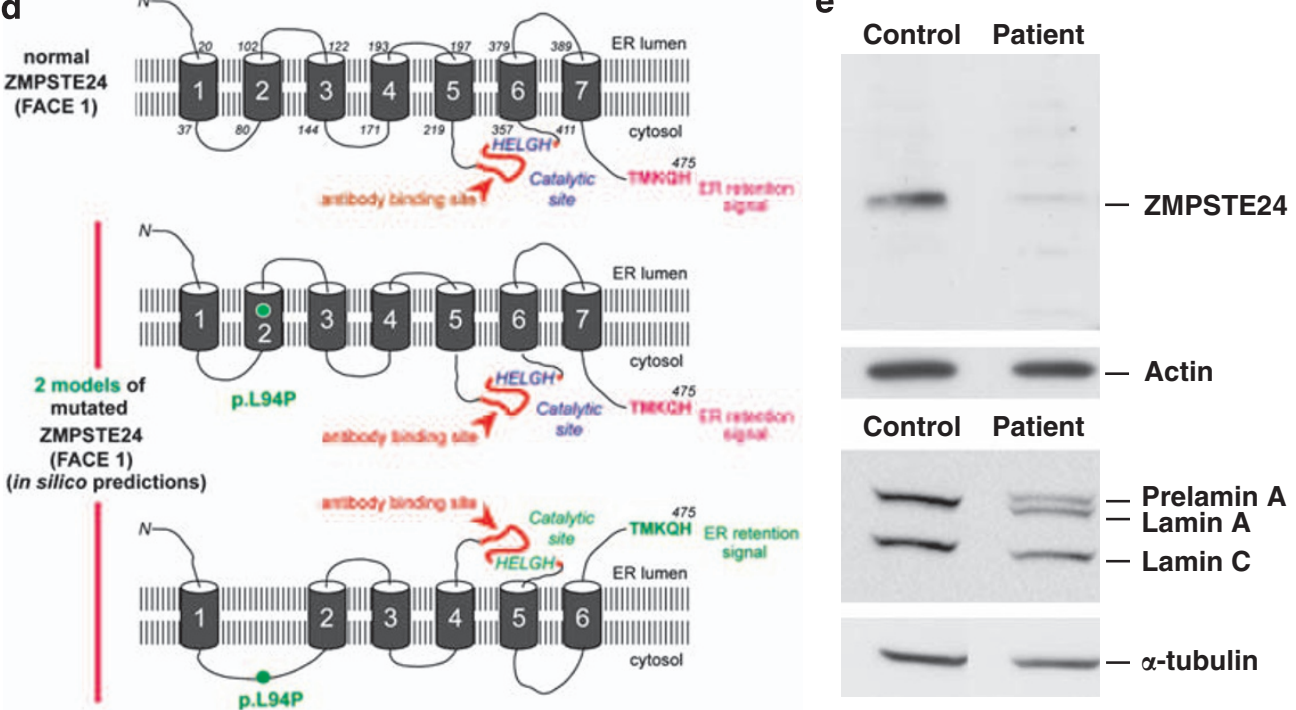

Figure 2 Characterization of the ZMPSTE24 mutation. (a) Genomic DNA sequences of patient and control showing the homozygous transition c.281T>C, p.Leu94Pro in ZMPSTE24 exon 3 gene. (b) Analysis of the segregation of the ZMPSTE24 variant within the family. Circles indicate females, squares indicate males, clear symbols indicate healthy subjects, black symbols indicate patients and ' $m$ ' indicates the mutated allele in each subject in the homozygous state $(\mathrm{m} / \mathrm{m})$ or the heterozygous state $(\mathrm{m} /+)$. The mutation creates a new Bs/l site in a 235-bp PCR fragment of exon 3. Bs/l digestion identifies two alleles: an uncut fragment indicates the wild-type allele; two additional bands indicate the mutated allele. (c) Partial amino acid sequence alignment of ZMPSTE24 from various species. The conservation of Leu94 is highlighted within the red box. (d) In silico predictions of the possible consequence of ZMPSTE24 mutation. TMHMM and TMpred programs, using either normal or mutated ZMPSTE24 protein sequences as matrix, indicated two possible conformation models. (e) Western blot analysis of ZMPSTE24 and A-type lamins at passage P2. The reduction of ZMPSTE24 in patient's cultured skin fibroblasts is associated with accumulation of prelamin A.

in-frame deletions sometimes resulting from splice site mutations ${ }^{10,11,16}$ in the ZMPSTE24 gene. Moreover, all RD-related mutations lead to a complete absence of the ZMPSTE24 protein or to null activity, consequently causing the accumulation of prelamin $\mathrm{A}$ and an absence of mature lamin A. ${ }^{11,16,25}$ Two patients carrying ZMPSTE24 mutations had progeroid features of variable severity. The first patient, carrying compound nonsense and missense mutations, had been diagnosed as a severe progeroid phenotype and died at the age of 2 years. ${ }^{14}$ The second patient carried a homozygous ZMPSTE24 frameshift deletion associated with an LMNA heterozygous nonsense mutation that 'rescued' the patient's phenotype, most probably by reducing to half the amounts of accumulated prelamin $\mathrm{A}$, as in the double-knockout Zmpste24 $4^{-/}$Lmna $^{+/-}$mice. ${ }^{15,31}$ This patient showed features compatible with a severe form of MADB, but was described as being affected with HGPS. ${ }^{15}$ In this last report, the authors concluded that the LMNA truncation is a salvage alteration modifying the clinical picture from RD to HGPS by preventing farnesylation of the accumulated prelamin A or by reducing the amount of accumulated prelamin A. This second hypothesis is more probable from our point of view, as no truncated lamin A isoform was visible on the western blot. In all cases, farnesylated prelamin A accumulated, whereas mature lamin A was either absent ${ }^{15}$ or reduced. ${ }^{14}$ Finally, five patients carrying compound heterozygous nonsense and missense ZMPSTE24 mutations have been described as being affected with MADB. ${ }^{9,32-34}$ These ZMPSTE24 mutations result not only in the presence of traces of prelamin A but also that 

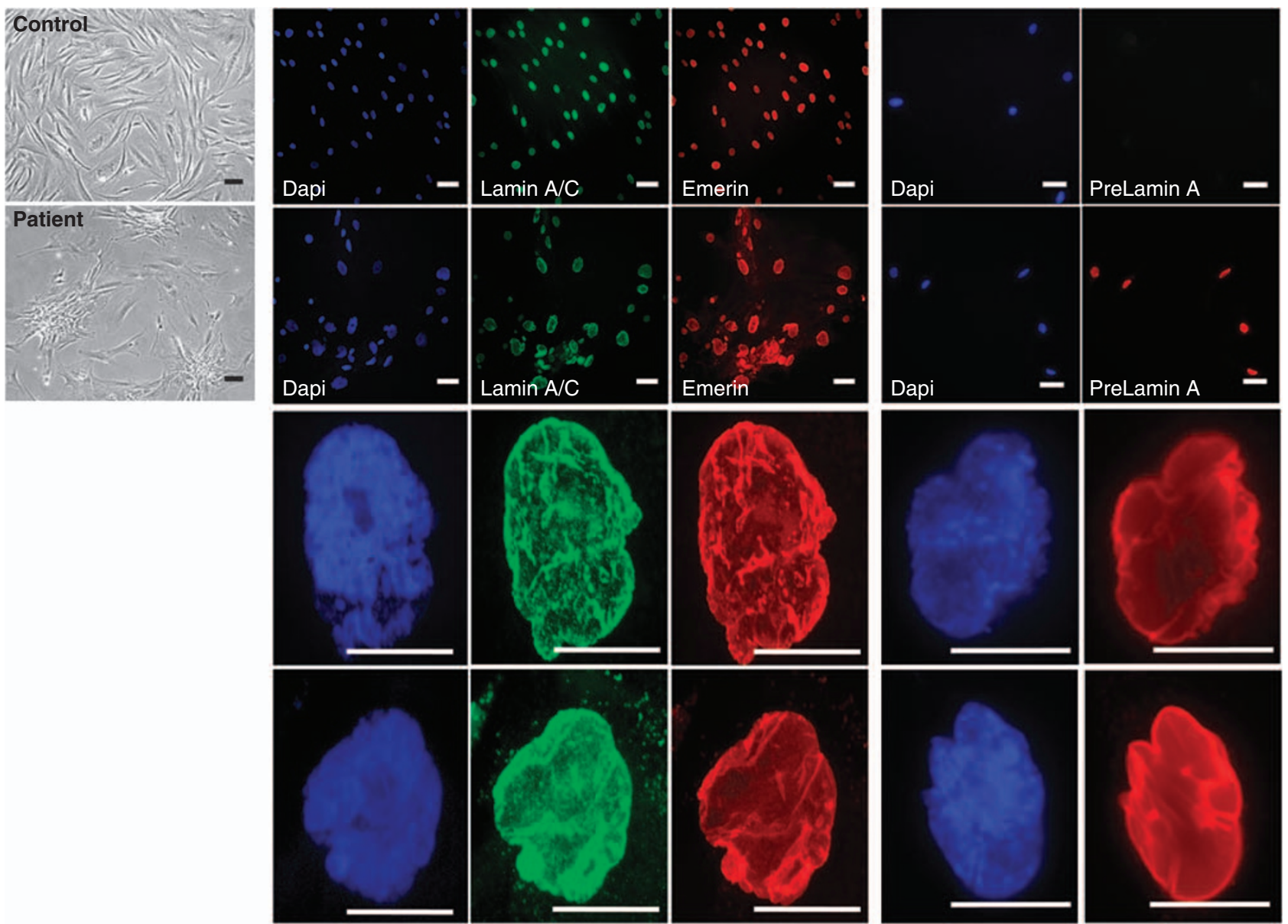

Figure $3 \mathrm{Immunofluorescence} \mathrm{analysis} \mathrm{of} \mathrm{the} \mathrm{patient's} \mathrm{fibroblasts.} \mathrm{Patient} \mathrm{fibroblasts} \mathrm{grow} \mathrm{as} \mathrm{clusters} \mathrm{and} \mathrm{exhibit} \mathrm{highly} \mathrm{variable} \mathrm{size} \mathrm{with} \mathrm{a} \mathrm{majority} \mathrm{of} \mathrm{the}$ cells being larger than control cells. Patient cells exhibited major nuclear shape defects, that is, lobulations, herniations, with lamins $A / C$ and emerin still localized at the nuclear rim. Prelamin A was present in all patient fibroblasts. Analyses were carried out at passage P2. Scale bar represents $20 \mu \mathrm{m}$.

of mature lamin $\mathrm{A}^{33}$ because of partial residual ZMPSTE24 activity. ${ }^{9,32,33}$

All these reports strongly suggest a link between phenotype severity, prelamin A accumulation levels and ZMPSTE24 residual activity. Complete prelamin A accumulation with null ZMPSTE24 enzyme activity can be correlated to the most severe progeroid syndrome, RD; null ZMPSTE24 activity rescued by a heterozygous LMNA null mutation causes a severe, HGPS-like, MADB phenotype, whereas partial accumulation of prelamin A with reduced ZMPSTE24 enzyme activity is associated with milder MADB phenotypes.

The patient reported here during the 30-year follow-up developed a $\mathrm{MADB}$ phenotype associated with a congenital myopathy, further extending the spectrum of clinical features associated with ZMPSTE24 mutations. Furthermore, our patient's MADB phenotype seems to recapitulate, to some extent, the main features of RD. Intrauterine growth retardation, tight skin and arthrogryposis at birth are classical features of RD. The disease evolved progressively and the whole clinical phenotype such as failure to thrive, mandible and clavicle hypoplasia, distal acro-osteolysis and scleroderma-like skin with subcutaneous calcifications appeared during the first and second decades, followed by vascular complications including high blood pressure and atherosclerosis as observed in other MADB patients. To our knowledge, this is the first report describing congenital myopathy in a patient affected with MADB.
The relatively mild progeroid phenotype of our patient is well correlated with the western blot analyses, which showed highly reduced expression of ZMPSTE24, with residual activity, as reduced levels of mature lamin A and partial prelamin A accumulation coexist in the patient's cells. In addition, according to the in silico studies, which predicted two conformational states of the mutated ZMPSTE24, we can imagine that one part of the mutated ZMPSTE24 is correctly folded despite the presence of mutation and is still functional, allowing normal maturation of prelamin A, whereas the other part missing the second TM domain and sharing enzymatic site and ER signal in the ER lumen is either degraded or unable to cleave prelamin A. The existence of both conformations could explain both the reduced level of ZMPSTE24 and the partial prelamin A maturation detected by western blot.

At the cellular level, the coexistence of both prelamin A and lamins $\mathrm{A}$ and $\mathrm{C}$ leads to a reduced lifespan of the cells with major nuclear abnormalities. ${ }^{10,35}$

From a phenotypic point of view, our patient suffered from particular features of prognostic value, including impressive subcutaneous and vascular calcifications. The unusual paravertebral diffuse calcinosis led to spinal cord compression, requiring surgery. Such calcifications have been reported in four ZMPSTE24-related MADB cases, two of them requiring surgical removal, ${ }^{9,36}$ but not in the two others. ${ }^{33}$ Similarly, a homozygous LMNA mutation c.1718C $>$ T leading 


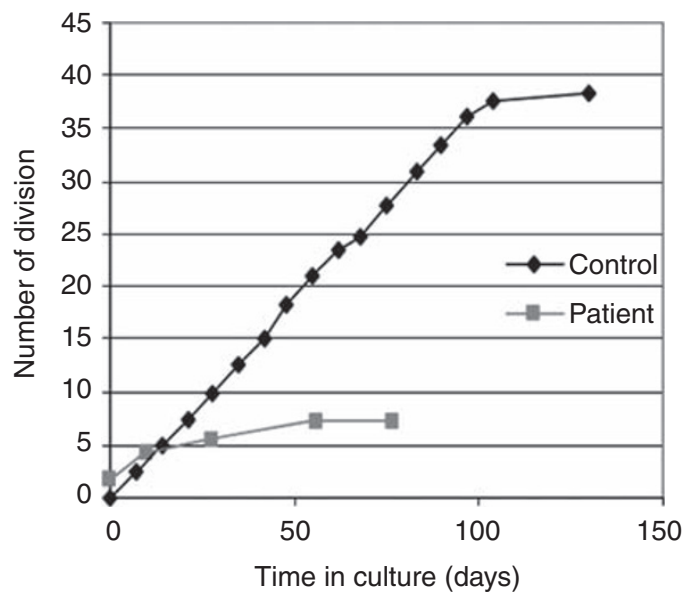

Figure 4 In vitro lifespan of fibroblasts. Cell populations were serially passaged in growth medium until the cells ceased to divide. At each passage the mean number of population doublings was determined by counting the cells. Patient fibroblasts showed a dramatically decreased replicative potential compared with cells isolated from a health control.

to the missense substitution p.Ser573Leu has been reported to result in progeroid features associated with arthropathy and tendinous calcinosis ${ }^{37}$ without any prelamin A accumulation. Furthermore, renal artery calcifications and chronic kidney disease were responsible for pharmacoresistant high blood pressure as observed in ZMPSTE24related MADB. ${ }^{32}$ The glomerular and tubular abnormalities observed in our patient argue for a multifactorial etiology.

In addition to progeroid features, our patient developed a clear and proven myopathy. She showed evidence of skeletal muscle involvement, including muscle weakness with scapular winging, myopathic EMG pattern and fiber-type disproportion with a predominance of small type I fibers and early respiratory insufficiency. These findings were unusual in the context of a ZMPSTE24-related progeroid syndrome. Skeletal muscle abnormalities, including muscle atrophy, first proximally (scapulae, buttocks) then distally in the limbs, are classical symptoms described in HGPS. ${ }^{38}$ As the diminished joint mobility observed in HGPS patients does not appear to be due to tightening of the skin, one may hypothesize that it may be due to muscle sclerosis and tightness similar to what is observed in myopathies. The emergence of LMNA gene mutations in HGPS patients prompted the examination of skeletal muscle, leading to the description of the first cases with progeroid syndrome and confirmed earlyonset myopathy due to p.Ser143Phe LMNA substitution ${ }^{39,40}$ or type A MAD due to p.Arg471Cys homozygous ${ }^{41}$ or p.Arg527His/Val440Met compound heterozygous ${ }^{42}$ LMNA mutation. Among the 30 previously reported ZMPSTE24-mutated patients; none had an obvious or proven skeletal muscle involvement. Although in our patient the first symptoms linked to muscle involvement appeared at 2.5 years, $\mathrm{RD}$ patients never reach this age and usually die in the first h/days after birth. Interestingly, even if none of the five ZMPSTE24-related MADB cases ${ }^{32-34}$ showed such myopathic involvement, one of these cases (patient 1 in Miyoshi et $a^{33}$ ) showed clear skeletal muscle fatty infiltration in gluteal and thigh muscles similar to what is described in other muscle diseases. On the other hand, Zmpste24-knockout mice $^{18,19}$ are normal at birth but show slightly variable phenotypes. Skeletal muscle involvement manifested mainly by abnormal gait and muscle weakness with either a normal histological aspect ${ }^{19}$ or a significant increase in abnormal small round fibers. ${ }^{18}$ The myopathic signs in our patient were similar to those observed in Zmpste24knockout mice. The etiopathogenic mechanism of skeletal muscle involvement in our patient remains to be determined. Unfortunately, muscle cells of the patient could not be explored; however, even if we cannot rule out the possibility that muscle disease is linked to one or more modifier genes, we hypothesize that they may exhibit reduced lifespan because of prelamin A accumulation, as do fibroblasts. Further skeletal muscle explorations in patients affected with MADB due to ZMPSTE24 loss of function might help to clarify this issue.

\section{CONFLICT OF INTEREST}

The authors declare no conflict of interest.

\section{ACKNOWLEDGEMENTS}

We thank Jean-Paul Leroy for his help in the clinical investigation of the patient, Thomas Voit for critical reading and fruitful discussion and the Myocastor study group for its constant support. This work was financially supported by the Institut National de la Santé et de la Recherche Médicale; the Université Pierre et Marie Curie Paris 06; the Université de la Méditerranée, the Centre National de la Recherche Scientifique; the Association Française contre les Myopathies (no. 11057, no. 11034); the European Union Sixth (Euro-laminopathies no. 018690) and Seventh (Myoage no. 223576) Framework Programmes.

1 Kilic F, Johnson DA, Sinensky M: Subcellular localization and partial purification of prelamin $A$ endoprotease: an enzyme which catalyzes the conversion of farnesylated prelamin A to mature lamin A. FEBS Lett 1999; 450: 61-65.

2 Leung GK, Schmidt WK, Bergo MO et al: Biochemical studies of Zmpste24-deficient mice. J Biol Chem 2001; 276: 29051-29058.

3 Lutz RJ, Trujillo MA, Denham KS, Wenger L, Sinensky M: Nucleoplasmic localization of prelamin A: implications for prenylation-dependent lamin A assembly into the nuclear lamina. Proc Natl Acad Sci USA 1992; 89: 3000-3004.

4 Varela I, Pereira S, Ugalde AP et al: Combined treatment with statins and aminobisphosphonates extends longevity in a mouse model of human premature aging. Nat Med 2008; 14: 767-772.

5 Sinensky M, Fantle K, Trujillo M, McLain T, Kupfer A, Dalton M: The processing pathway of prelamin A. J Cell Sci 1994; 107 (Part 1): 61-67.

6 Corrigan DP, Kuszczak D, Rusinol AE et al: Prelamin A endoproteolytic processing in vitro by recombinant Zmpste24. Biochem J 2005; 387: 129-138.

7 Broers J, Ramaekers F, Bonne G, Ben Yaou R, Hutchison C: The nuclear lamins: laminopathies and their role in premature ageing. Physiological Reviews 2006; 86: 967-1008.

8 Worman HJ, Bonne G: 'Laminopathies': a wide spectrum of human diseases. Exp Cell Res 2007; 313: 2121-2133.

9 Agarwal AK, Fryns JP, Auchus RJ, Garg A: Zinc metalloproteinase, ZMPSTE24, is mutated in mandibuloacral dysplasia. Hum Mol Genet 2003; 12: 1995-2001.

10 Navarro C, De Sandre-Giovannoli A, Bernard R et al: Lamin A and ZMPSTE24 (FACE-1) defects cause nuclear disorganisation and identify restrictive dermopathy as a lethal neonatal laminopathy. Hum Mol Genet 2004; 13: 2493-2503.

11 Navarro CL, Cadinanos J, Sandre-Giovannoli AD et al: Loss of ZMPSTE24 (FACE-1) causes autosomal recessive restrictive dermopathy and accumulation of Lamin A precursors. Hum Mol Genet 2005; 14: 1503-1513.

12 Witt DR, Hayden MR, Holbrook KA, Dale BA, Baldwin VJ, Taylor GP: Restrictive dermopathy: a newly recognized autosomal recessive skin dysplasia. Am J Med Genet 1986; 24: 631-648.

13 Novelli G, Muchir A, Sangiuolo F et al: Mandibuloacral dysplasia is caused by a mutation in LMNA encoding lamins A/C. Am J Hum Genet 2002; 71: 426-431.

14 Shackleton S, Smallwood DT, Clayton P et al: Compound heterozygous ZMPSTE24 mutations reduce prelamin A processing and result in a severe progeroid phenotype. J Med Genet 2005; 42: e36.

15 Denecke J, Brune T, Feldhaus T et al: A homozygous ZMPSTE24 null mutation in combination with a heterozygous mutation in the LMNA gene causes HutchinsonGilford progeria syndrome (HGPS): insights into the pathophysiology of HGPS. Hum Mutat 2006; 27: 524-531.

16 Sander CS, Salman N, van Geel M et al: A newly identified splice site mutation in ZMPSTE24 causes restrictive dermopathy in the Middle East. Br J Dermatol 2008; 159: 961-967.

17 Smigiel R, Jakubiak A, Esteves-Vieira $V$ et al: Novel frameshifting mutations of the ZMPSTE24 gene in two siblings affected with restrictive dermopathy and review of the mutations described in the literature. Am J Med Genet A 2010; 152A: 447-452. 
18 Pendas AM, Zhou Z, Cadinanos J et al: Defective prelamin A processing and muscular and adipocyte alterations in Zmpste24 metalloproteinase-deficient mice. Nat Genet 2002; 31: 94-99.

19 Bergo MO, Gavino B, Ross J et al: Zmpste24 deficiency in mice causes spontaneous bone fractures, muscle weakness, and a prelamin A processing defect. Proc Natl Acad Sci USA 2002; 99: 13049-13054.

20 Fong LG, Frost $\mathrm{D}$, Meta $\mathrm{M}$ et al: A protein farnesyltransferase inhibitor ameliorates disease in a mouse model of progeria. Science 2006; 311: 1621-1623.

21 Ben Yaou R, Toutain A, Arimura T et al: Multitissular involvement in a family with LMNA and EMD mutations: role of digenic mechanism? Neurology 2007; 68: 1883-1894.

22 Muchir A, Medioni J, Laluc M et al: Nuclear envelope alterations in fibroblasts from patients with muscular dystrophy, cardiomyopathy, and partial lipodystrophy carrying lamin A/C gene mutations. Muscle Nerve 2004; 30: 444-450.

23 Decary S, Mouly V, Hamida CB, Sautet A, Barbet JP, Butler-Browne GS: Replicative potential and telomere length in human skeletal muscle: implications for satellite cell-mediated gene therapy. Hum Gene Ther 1997; 8: 1429-1438.

24 Thill M, Nguyen TD, Wehnert $M$ et al: Restrictive dermopathy: a rare laminopathy. Arch Gynecol Obstet 2008; 278: 201-208.

25 Moulson CL, Go G, Gardner JM et al: Homozygous and compound heterozygous mutations in ZMPSTE24 cause the laminopathy restrictive dermopathy. J Invest Dermatol 2005; 125: 913-919.

26 Morais P, Magina S, Ribeiro Mdo C et al: Restrictive dermopathy-a lethal congenital laminopathy. case report and review of the literature. Eur J Pediatr 2009; 168: 1007-1012.

27 Kariminejad A, Goodarzi P, Thanh Huong le T, Wehnert MS: Restrictive dermopathy. Molecular diagnosis of restrictive dermopathy in a stillborn fetus from a consanguineous Iranian family. Saudi Med J 2009; 30: 150-153.

28 Chen M, Kuo HH, Huang YC et al: A case of restrictive dermopathy with complete chorioamniotic membrane separation caused by a novel homozygous nonsense mutation in the ZMPSTE24 gene. Am J Med Genet A 2009; 149A: 1550-1554.

29 Jagadeesh S, Bhat L, Suresh I, Muralidhar SL: Prenatal diagnosis of restrictive dermopathy. Indian Pediatr 2009; 46: 349-351.

$30 \mathrm{Li}$ C: Homozygosity for the common mutation c.1085dupT in the ZMPSTE24 gene in a Mennonite baby with restrictive dermopathy and placenta abruption. Am J Med Genet A 2010; 152A: 262-263.
31 Fong LG, Ng JK, Meta M et al: Heterozygosity for Lmna deficiency eliminates the progeria-like phenotypes in Zmpste24-deficient mice. Proc Natl Acad Sci USA 2004; 101: 18111-18116.

32 Agarwal AK, Zhou XJ, Hall RK et al: Focal segmental glomerulosclerosis in patients with mandibuloacral dysplasia owing to ZMPSTE24 deficiency. J Investig Med 2006; 54: 208-213.

33 Miyoshi Y, Akagi M, Agarwal AK et al: Severe mandibuloacral dysplasia caused by novel compound heterozygous ZMPSTE24 mutations in two Japanese siblings. Clin Genet 2008; 73: 535-544.

34 Cunningham VJ, D'Apice MR, Licata N, Novelli G, Cundy T: Skeletal phenotype of mandibuloacral dysplasia associated with mutations in ZMPSTE24. Bone 2010; 47: 591-597.

35 Goldman RD, Shumaker DK, Erdos MR et al: Accumulation of mutant lamin A causes progressive changes in nuclear architecture in Hutchinson-Gilford progeria syndrome. Proc Natl Acad Sci USA 2004; 101: 8963-8968.

36 Agarwal AK, Kazachkova I, Ten S, Garg A: Severe mandibuloacral dysplasia-associated lipodystrophy and progeria in a young girl with a novel homozygous Arg527Cys LMNA mutation. J Clin Endocrinol Metab 2008; 93: 4617-4623.

37 Van Esch H, Agarwal AK, Debeer P, Fryns JP, Garg A: A homozygous mutation in the lamin $A / C$ gene associated with a novel syndrome of arthropathy, tendinous calcinosis, and progeroid features. J Clin Endocrinol Metab 2006; 91: 517-521.

38 Hennekam RC: Hutchinson-Gilford progeria syndrome: review of the phenotype. Am J Med Genet A 2006; 140: 2603-2624.

39 Kirschner J, Brune $T$, Wehnert $\mathrm{M}$ et al: p.S143F mutation in lamin $\mathrm{A} / \mathrm{C}$ : a new phenotype combining myopathy and progeria. Ann Neurol 2005; 57: $148-151$.

40 Madej-Pilarczyk A, Kmiec T, Fidzianska A et al: Progeria caused by a rare LMNA mutation p.S143F associated with mild myopathy and atrial fibrillation. Eur J Paediatr Neurol 2008; 12: 427-430.

41 Zirn B, Kress W, Grimm T et al: Association of homozygous LMNA mutation R471C with new phenotype: mandibuloacral dysplasia, progeria, and rigid spine muscular dystrophy. Am J Med Genet A 2008; 146A: 1049-1054.

42 Lombardi F, Gullotta F, Columbaro $\mathrm{M}$ et al: Compound heterozygosity for mutations in LMNA in a patient with a myopathic and lipodystrophic mandibuloacral dysplasia type A phenotype. J Clin Endocrinol Metab 2007; 92: 4467-4471. 